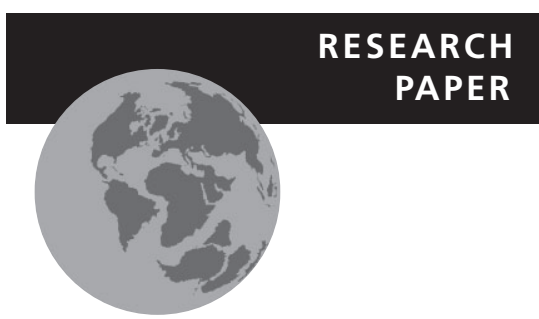

\section{Beyond climate: disturbance niche shifts in invasive species}

\author{
Pablo González-Moreno ${ }^{1 \star}$, Jeffrey M. Diez ${ }^{2,3}$, David M. Richardson ${ }^{4}$ and \\ Montserrat Vilà ${ }^{1}$
}

\begin{abstract}
Aim Analysing how species niches shift between native and introduced ranges is a powerful tool for understanding the determinants of species distributions and for anticipating range expansions by invasive species. Most studies only consider the climatic niche, by correlating widely available presence-only data with regional climate. However, habitat characteristics and disturbance also shape species niches, thereby potentially confounding shifts attributed only to differences in climate. Here we used presence and abundance data for Oxalis pes-caprae, a species native to South Africa and invading areas globally, to understand how niche shifts may be influenced by disturbance at habitat and landscape scales in addition to climate.
\end{abstract}

Locality Mediterranean climate areas world-wide.

Methods We used available presence-only data and also conducted extensive surveys of the abundance of Oxalis (c. 11,000 plots) across different habitats in South Africa and in the introduced range in the Mediterranean Basin. We extended principal component analysis methods for measuring niche shifts by using Bayesian generalized linear models to identify climatic and disturbance niche shifts.

Results We found a large climatic niche expansion towards stronger seasonality and lower temperature in the introduced range, but this expansion was greatly reduced when considering only conditions available in both ranges. Oxalis occupied more natural landscapes in the native range that remained unoccupied in the introduced range ('niche unfilling'). In contrast to the similar abundances in natural and disturbed habitats in its native range, Oxalis was more abundant in disturbed habitats in the introduced range.

Conclusions The large climatic niche expansion most likely reflects significant plasticity of Oxalis rather than rapid evolution. Furthermore, the unfilling of its disturbance niche in the introduced range suggests high potential for further invasion of natural areas. Together, these findings suggest that the potential for future spread of invasive species may be underestimated by approaches that characterize species niches based only on climate or partial information about their distributions.

\section{Keywords}

Bayesian, biological invasions, invasion risk, niche conservatism, non-native species, Oxalis pes-caprae, reciprocal distribution modelling.

\section{INTRODUCTION}

Understanding the determinants of invasive species distributions is crucial for effective management. A common approach is to make predictions about the potential introduced range using the data about the species' distribution in its native or introduced range (Peterson et al., 2003; Thuiller et al., 2005). This approach relies on the assumption that species have similar niche characteristics in native and introduced ranges (i.e. niche conservatism) (Peterson et al., 2003; Alexander \& Edwards, 2010). Recent studies have questioned this assumption for plants (Broennimann et al., 2007; Gallagher et al., 2010), insects 
(Fitzpatrick et al., 2007; Medley, 2010), amphibians (Urban et al., 2007) and fish (Lauzeral et al., 2011) by highlighting the potential importance of rapid evolution and altered biotic interactions during the invasion process. Nevertheless, species may only have access to a subset of the potentially suitable climatic conditions in their native ranges. Consequently, when analyses of climatic niche shifts are limited to those climates available in both native and introduced ranges (i.e. analogous conditions) there appear to be fewer real niche shifts (Petitpierre et al., 2012).

Knowing whether climatic niches are similar in the native and introduced range is extremely important for predicting species occurrences in new areas and in response to climate change (Alexander \& Edwards, 2010). For example, observed shifts into novel climates in the introduced range that are not occupied in the native range (i.e. expansion) suggest that rapid evolution may have expanded a species' climatic tolerances. Conversely, narrower climatic envelopes observed in the introduced range than in the native range (i.e. unfilling) suggest the importance of dispersal limitation, lag phases and/or evolutionary bottlenecks associated with introduction processes (Medley, 2010; Guisan et al., 2014). Furthermore, some processes, such as changes in biotic interactions between the ranges, may lead to either niche expansion or unfilling. Quantifying the prevalence of niche unfilling in the invaded range is critically important because it suggests the likelihood of further spread over time in the introduced range.

Niche conservatism in invasive species is typically tested using broad climatic niches, despite the fact that similar ideas may be applied to other aspects of a species' niche, such as disturbance regimes or habitat characteristics (Wiens et al., 2010; Guisan et al., 2014). For example, many invasive plant species are favoured by the nutrient-rich conditions provided by high levels of disturbance (Hobbs \& Huenneke, 1992). It has been suggested that non-native species tend to colonize disturbed habitats in the early stages of the invasion process, and may then expand to more natural conditions as the invasion unfolds (Dietz \& Edwards, 2006). The association of a species with disturbance (i.e. disturbance niche) may be related to local gradients from natural to ruderal habitats (Vilà et al., 2007), and also human alterations at the landscape scale (González-Moreno et al., 2013). Although theory suggests a shift towards more altered habitats and landscapes in the introduced range, the roles of habitat and disturbance in mediating niche shifts have received much less attention than climatic niche shifts. Nonetheless, it is important to understand shifts in a species' disturbance niche because they may contribute to the time lags that often characterize invasions (Geerts et al., 2013).

In this study we used Oxalis pes-caprae L., a geophyte native to South Africa which has invaded Mediterranean climate areas globally, to test for shifts in both climatic and disturbance niches between native and introduced areas. First, we quantified the climatic niche conservatism of the species using global data (30' resolution) and ordination methods. Second, we examined the climatic and disturbance niches using data on the abundance of the species from extensive field sampling (c. 11,000 plots; 30" resolution) throughout the native range in South Africa and across large parts of the introduced range in the Mediterranean Basin. We hypothesized that different niche shifts may be apparent when considering abundance compared with the widely used occurrence data. We used a combination of principal components analysis (PCA) methods and generalized linear models (GLMs) within a Bayesian framework to provide a more detailed test of how the species' climate and disturbance niches shift in the introduced region. We interpret the results to provide guidelines for more accurate modelling of invasive species and consider implications for the potential invasion of Oxalis into natural areas in the long term.

\section{METHODS}

\section{Study species}

Oxalis pes-caprae L. (hereafter Oxalis) is a small geophyte from South Africa which has invaded many Mediterranean areas globally (Weber, 2003). It was introduced to the Mediterranean Basin in 1796, and reached mainland Spain by 1825 and the Balearic Islands by 1870 (D'Austria, 1884). Despite its long history in the Mediterranean Basin, in Spain it only covers 46\% of its potential distribution (Gassó et al., 2012). It generally flowers from late autumn to early summer and is heterostylic with three flower morphs (Castro et al., 2007). In its native range all three floral morphs occur and the species reproduces both sexually and asexually (Ornduff, 1987), while in the introduced range only one morph is dominant and the principal means of reproduction is via vegetative bulbils (Vilà et al., 2006; Castro et al., 2007).

\section{Global climatic niche shift analyses}

\section{Plant dataset}

We collected all available presence-only data for Oxalis in the Global Biodiversity Information Facility (1818 plots; Fig. 1); this provided good coverage for the native range (South Africa) and a large part of the introduced range (North America, Europe and Australia). We kept those plots with at least $0.001^{\circ}$ positional accuracy and excluded isolated locations outside its known distribution (most likely botanic gardens). In addition, we added 1852 occurrence plots from our surveys in South Africa and Europe (see below).

\section{Climate data}

We extracted 19 bioclimatic variables from WorldClim (Hijmans et al., 2005) at 30' resolution (c. $50 \mathrm{~km}$ ). We included as explanatory variables only the minimum combination of climatic variables identified as not collinear within each range (Table S1 in Appendix S1 in the Supporting Information): mean annual temperature, temperature seasonality, precipitation seasonality, winter precipitation (coldest quarter), minimum 


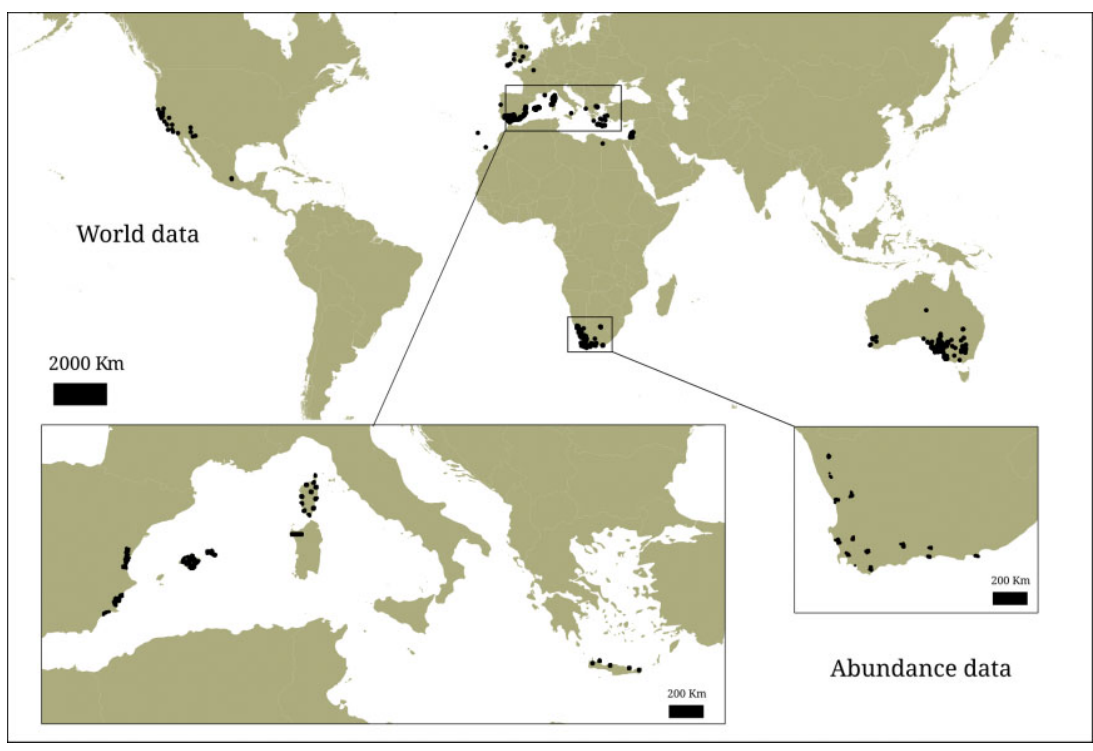

Figure 1 Oxalis pes-caprae plots used in the analyses. The upper part of the figure shows the localities of presence-only data used to test the global climatic niche shift. The panels in the lower half show the localities of plots where abundance data were collected in the introduced range (Mediterranean Basin, left) and in the native range (South Africa, right). temperature of the coldest month (minimum temperature) and temperature of the driest quarter.

\section{Statistical analyses}

We identified climatic niche shifts for Oxalis between native and introduced ranges within the whole Mediterranean biome using the global occurrence data and the climatic data at 30' resolution. To avoid pseudo-replication we included only one plot for each $30^{\prime} \times 30^{\prime}$ grid cell ( 342 plots). Our analysis of niche shifts followed the three-step approach used by Broennimann et al., (2012) and Petitpierre et al., (2012). First, we delimited the Mediterranean biome (Olson et al., 2001) for both ranges and extracted all climate data at $30^{\prime}$ resolution. Based on these data, we performed a PCA and used its first two axes to characterize the environmental space within which we could compare the native and introduced niches (Fig. S1 in Appendix S1). We then divided this environmental space into a grid of $100 \times 100$ cells, as in Broennimann et al. (2012). Second, we used a kernel density function to convert occurrences of Oxalis and the available climates in each range into densities in order to correct for sampling bias and environmental availability, respectively, and to ensure that the results were independent of the grid resolution (Broennimann et al., 2012). Third, we calculated three indices of niche shifts: unfilling, stability and expansion (Petitpierre et al., 2012) considering either the common environmental space for native and introduced ranges or the whole environmental space. Unfilling is defined as the proportion of the densities in the native range located in different conditions from the introduced range, stability is the proportion of the densities in the introduced range that overlap with the native range, and expansion is the proportion of the densities in the introduced range located in different conditions from the native range. We calculated each index using the 75th percentile of the available environmental conditions in each range. This approach removes the marginal environments and avoids bias due to artefacts of the density function (Petitpierre et al., 2012). Finally, we calculated the median of the distribution density and the median of the available climatic space in both ranges in order to assess the overall direction of the shifts.

\section{Mediterranean Basin niche shift analyses}

\section{Plant dataset}

We obtained local abundance data in the introduced range from two different datasets in the Mediterranean Basin: from Gimeno et al. (2006), conducted on two Balearic islands (Mallorca and Menorca) and two adjacent coastal regions of mainland Spain (Valencia and Murcia), and from Affre et al. (2010), conducted on three Mediterranean islands (Crete, Sardinia and Corsica). The two datasets were originally sampled using similar protocols.

1. Five $10 \mathrm{~km} \times 10 \mathrm{~km}$ Universal Transverse Mercator (UTM) cells were randomly chosen in each region.

2. Within each UTM cell 100 plots were sampled. In Gimeno et al. (2006) exact survey plots were selected by stopping every $1 \mathrm{~km}$ along several communication networks (from main roads to paths). In contrast, in Affre et al. (2010) the 100 plots were randomly located (GPS accuracy $<10 \mathrm{~m}$ ). Accessibility problems during sampling finally lead to fewer than 100 plots per block, so the final dataset included 1716 plots from Gimeno et al. (2006) and 1355 from Affre et al. (2010).

3. In each plot, dominant habitats falling within a 50-m radius were classified according to ten categories that varied in their level of human influence (i.e. disturbance at the local scale) agriculture, coastal, forest, grassland, river, transport network (road and railway margins), rocky, ruderal (disturbed open areas), shrubland and urban (built areas) - resulting in one or more data entries for each sampled plot.

For all habitats surveyed at each plot, Oxalis cover was categorized using a six-point index based on percentage cover: 
dominant $(\geq 75 \%)=5$, abundant $(75-50 \%)=4$, frequent $(50$ $25 \%)=3$, occasional $(25-5 \%)=2$, rare $(\leq 5->0 \%)=1$ and absent $(0 \%)=0$.

To characterize the distribution of Oxalis in its native range we followed the protocol by Gimeno et al. (2006). We sampled $1310 \mathrm{~km} \times 10 \mathrm{~km}$ UTM cells (1033 plots) covering the whole gradient of climatic conditions where Oxalis occurs (as indicated by experts and herbarium data). We also considered the gradient of human influence by placing the grids close to urban nuclei of different sizes.

\section{Climate and landscape data}

As for the global analysis we extracted 19 bioclimatic variables from WorldClim for all plots, but at 30" resolution (c. $1 \mathrm{~km})$. To avoid collinearity problems in the regression analyses we selected only poorly correlated variables $(r<0.7)$ : mean annual temperature, temperature seasonality, precipitation seasonality and winter precipitation.

To characterize human influence at the landscape scale for each plot we obtained the following land-cover variables (percentage of urban, agricultural and natural land covers), minimum distance to roads and human footprint index. Landcover data were obtained from the European CORINE landcover map (2006, Crete from 2000) and the South African NLC2009. We resampled raster maps to $100-\mathrm{m}$ resolution (minimum resolution of the CORINE map) and calculated land-cover percentage at three buffer distances from each plot: 250, 500 and $1000 \mathrm{~m}$. Road network maps were obtained from the global community-owned project OpenStreetMap. This dataset is highly accurate compared with corporate datasets (Neis et al., 2011) and we assume a similar error for all study areas. Finally, index data on the human footprint were extracted from the Last of the Wild Project at 1-km resolution (WCS \& CIESIN, 2005). Based on collinearity analyses we selected agricultural and urban land covers, distance to roads and human footprint variables (Table S1 in Appendix S1). We identified $1000 \mathrm{~m}$ as the most appropriate buffer distance based on Pearson's correlations between the abundance of Oxalis and land-cover variables across distances (Table S2 in Appendix S1). All data extraction and manipulation was done using opensource GIS software (QGIS, GRASs and POSTGIS).

\section{Statistical analyses}

We used ordination (PCA) and GLMs to test climate and disturbance niche conservatism of Oxalis between the native range (4862 data entries considering the habitats found in each of the 1033 plots) and across a large part of the introduced range in the Mediterranean Basin (6009 data entries corresponding to 3071 plots). Ordination allowed for a comparison of species niches for each range in a space defined by axes summarizing several variables. We used GLMs to compare the changes between ranges in the association of Oxalis abundance with each variable and in the difference in local habitat suitability.

\section{Ordination method}

We used the same PCA approach as in the global analysis to quantify climatic and disturbance niche shifts between the introduced range in the Mediterranean Basin and the native range in South Africa. We compared the niches for both ranges using all climatic (30" resolution) and disturbance (landscape scale) conditions as environmental space for all plots sampled, irrespective of the occurrence of Oxalis. With this approach we assumed a good coverage of the available environmental space in the native range but it covers only a restricted portion of the introduced range in the Mediterranean Basin. For the PCA we used the same climate variables as in the global analysis. However, we used those landscape variables selected by collinearity analyses plus natural land cover as the disturbance variables. The collinearity among variables does not affect the results, and facilitates better distinction of the disturbance gradient. We analysed shifts using both presence and abundance data. For the abundance data, we adapted the method by replicating plots according to their level of abundance (1 to 5). This weights the species density by its abundance, such that areas of the PCA where Oxalis shows higher abundance will proportionally include higher species density.

\section{Modelling shifts using GLMs}

We identified how climate and disturbance (habitat and landscape scales) affected shifts in the abundance of Oxalis using Bayesian models that allowed regression coefficients to vary between the native and introduced ranges. The abundance of Oxalis in the dominant habitats of each plot $(n=10,871)$, was modelled using a binomial distribution considering climate ( $30^{\prime \prime}$ resolution) and landscape variables that were not correlated for both ranges (for details see Appendix S2). We corrected for the potential sampling bias arising from the roadside collection of some data by including the distance to roads as a covariate. We also compared models that included only road sampling data with models including all data. Because results were very similar, we focus on results using all data ('road only' models in Appendix S1).

We calculated several aggregate indices based on the model coefficients to facilitate their interpretation. First, we calculated the overall suitability of disturbed habitats in each range as the sum of the coefficients of agricultural, transport network, urban and ruderal habitats. Similarly, we calculated the overall suitability of natural habitats for each range as the sum of the coefficients for coastal, forest, grasslands, river, rocky and shrubland habitats. Finally, to quantify the overall importance of disturbance variables at the landscape scale in each range we calculated the sum of the absolute values of their coefficients.

We set apart a randomly selected $10 \%$ of plots for model validation and calibrated the models with the remaining $90 \%$. Goodness of fit of the models was calculated using deviance information criteria (DIC) (Spiegelhalter et al., 2002) for the whole model and mean squared errors (predicted minus observed) of the validation plots for each range. Models that 
minimized both values were considered to fit the data better. In preliminary analyses, full models without quadratic terms were always equivalent to more simple models (within 2-4 DIC). Thus, we ran final models using all climate and landscape variables selected by collinearity analyses without quadratic terms. We did not detect significant spatial autocorrelation in the residuals of the models at bins of $0.1^{\circ}$ (Moran's index $<0.1$ ). Final models were run in OpenBUGS 3.2.1 (Lunn et al., 2009) until convergence of the parameters was ensured (c. 25,000 iterations, convergence assessed visually), after which the parameters were calculated from another 25,000 iterations (code in Appendix S2).

\section{Predicting shifts}

To visually compare the distribution of Oxalis in native and introduced ranges we made predictions of the abundance of the species in southern Europe using GLMs calibrated with either native or introduced data (reciprocal distribution modelling; Medley, 2010). We used the posterior parameter values of the best model to generate mean predictions at the central point of 1-km UTM grid resolution, irrespective of the habitat type. We also identified non-analogous conditions to the calibrated data based on the multivariate environmental similarity surfaces (MESS) index (Elith et al., 2010) where models are extrapolating and predictions should be interpreted cautiously (Fitzpatrick \& Hargrove, 2009).

\section{RESULTS}

\section{Global climatic niche shift}

The climatic space of the whole Mediterranean biome was summarized by two PCA axes explained mainly by minimum temperature (36.9\%) and precipitation seasonality (32.2\%) (Fig. S1 in Appendix S1). Using this climatic space we found that the median of the distribution of Oxalis in the introduced range moved towards a lower temperature and slightly stronger seasonality relative to the native range (Fig. 2). More specifically, the climatic niche of Oxalis expanded into areas with both stronger and weaker precipitation seasonality, extending into some areas outside the climatic envelope of the Mediterranean biome (37.3\% expansion). On the other hand, some small areas with higher temperature and medium seasonality were only occupied in the native range (3.1\% unfilling). Taking into account only the common environmental space between the native and introduced range (overlap between black and grey lines in Fig. 2), expansion and unfilling were reduced in both cases to just $3.5 \%$ and $0 \%$ respectively.

\section{Mediterranean Basin niche shift}

\section{Comparing niche shifts in an ordination space}

The climatic space across all the sampled abundance plots was summarized by two axes corresponding mainly to precipitation seasonality (37.2\%) and mean annual temperature (28.5\%)

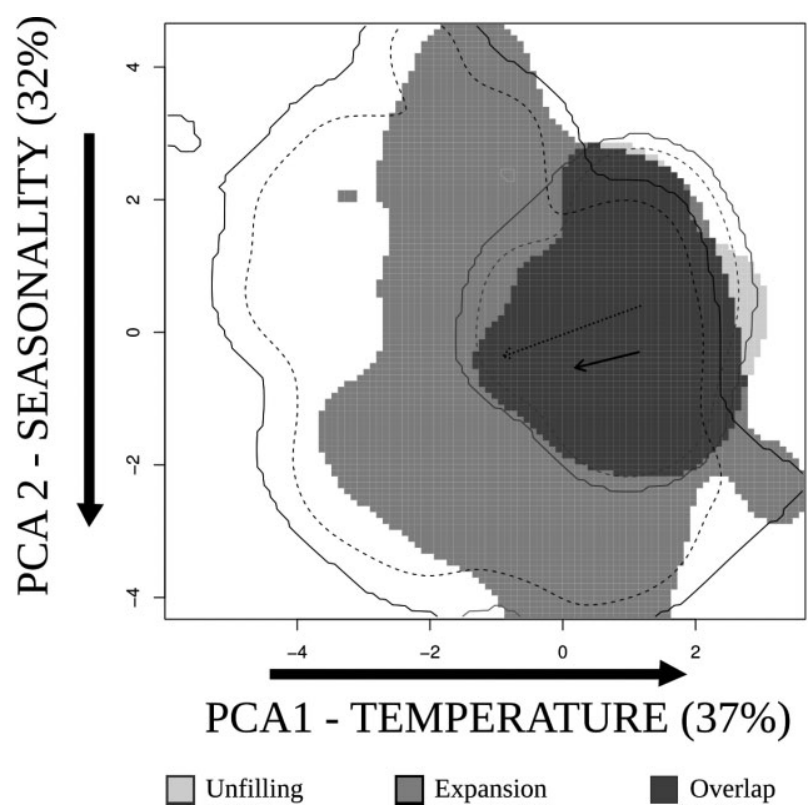

Figure 2 Global climatic niche shift of Oxalis pes-caprae based on a principal components analysis (PCA) of climate variables. The light grey line delimits the entire range of conditions in the native range of Oxalis while the black line delimits the conditions in the introduced range (the whole Mediterranean biome without considering South Africa). For both ranges, the continuous line corresponds to the whole climatic space while the dashed line indicates the 75 th percentile. The overlapped space between grey and black lines corresponds to the common environmental space between ranges. Shaded areas represent the portion of these conditions actually occupied by Oxalis: medium grey for niche expansion (presence only in the introduced range), light grey for niche unfilling (presence only in the native range) and dark grey for the conditions that are occupied in both ranges. The continuous black arrow shows the environmental distance between the median of the distribution density for each range. The dashed black arrow shows the environmental distance between the median of the available climatic space in each range. See Appendix S1 for detailed plots.

(Fig. S2 in Appendix S1). The median of the Oxalis distribution shifted towards stronger seasonality and slightly lower temperature in the introduced range (Fig. 3a). The climatic niche of the species was particularly expanded to areas with stronger precipitation seasonality occurring in Malta (15\% expansion). On the other hand, some areas with weaker seasonality were only occupied in the native range ( $68 \%$ unfilling). Taking into account the common environmental space, there was no expansion and unfilling was $10 \%$. Results considering only presence data were very similar but with lower percentages (4\% unfilling in the common space; Fig. S4 in Appendix S1). These results are very similar to the global climatic niche shift but with higher rates.

The disturbance space at the landscape scale was summarized by two axes corresponding to natural versus agricultural areas (43.2\%) and urban areas versus distance to roads (28.3\%) (Fig. S2 in Appendix S1). Using this space, we found a shift of the median of the Oxalis distribution in the introduced range 
A

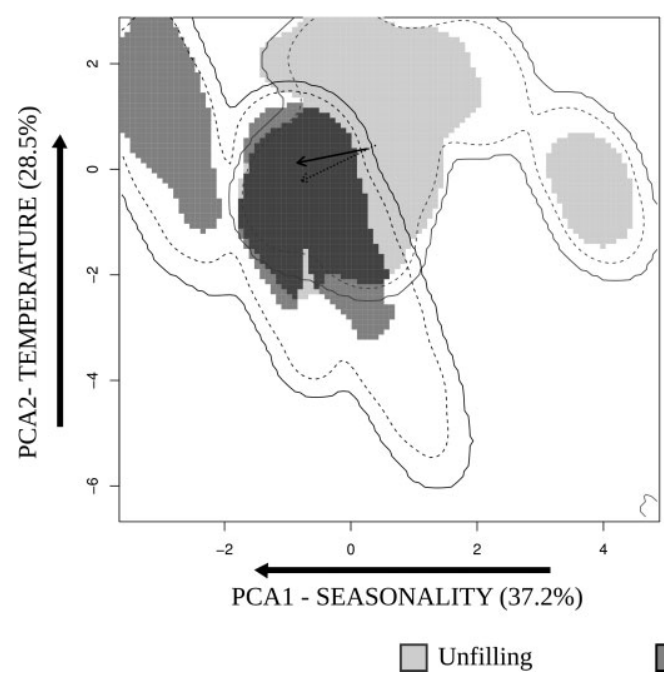

B

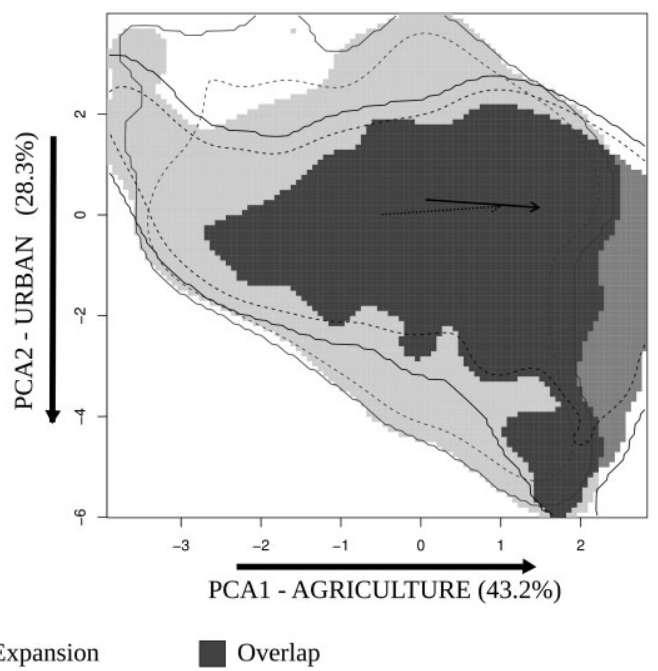

Figure 3 Shifts in the climatic and disturbance niche of Oxalis pes-caprae (abundance) between South Africa and the Mediterranean Basin based on separate principal components analyses (PCAs) for climate variables and disturbance variables at the landscape scale. See legend in Figure 2 and Appendix S1 for detailed plots.

towards more disturbed areas (Fig. 3b), but that corresponded to only $0.9 \%$ of expansion to more disturbed areas. This expansion occurred at a landscape configuration not available in the native range, corresponding to areas of intensive agriculture in eastern mainland Spain. Areas with the highest natural land cover were only occupied in the native range (15\% unfilling). When considering the common disturbance space, this unfilling was reduced to $8 \%$. Results considering only presence data were very similar, but with slightly lower percentages ( $6 \%$ unfilling in the common space; Fig. S4 in Appendix S1).

\section{Comparing niche shifts in a regression model}

The final model showed rather low errors for the native range compared with the introduced range (Table 1). Half of the climatic variables showed similar associations with the abundance of Oxalis in both ranges (Fig. 4). The abundance of Oxalis increased at higher mean annual temperature and stronger precipitation seasonality in both ranges. Nevertheless, precipitation in winter seems to be positively associated with abundance of Oxalis only in the native range, whereas temperature seasonality was negatively associated with abundance only in the introduced range.

Proximity to roads and agricultural and urban land-cover types were all positively associated with abundance of Oxalis in both ranges (Fig. 4). Furthermore, proximity to roads, urban cover and the human footprint index were all more strongly associated with abundance of Oxalis in the introduced range than in the native range (Fig. 4, Table S3 in Appendix S1). The overall difference for disturbance variables at the landscape scale was also significantly higher in the introduced range.

In the native range, abundance of Oxalis was rather similar across habitats, although ruderal and transport network habitats
Table 1 Mean and standard deviation (SD) of the squared errors (SE; predicted minus observed) in the models explaining the abundance of Oxalis (Mediterranean Basin niche shift analyses) for the validation plots set apart (10\%) for the native and introduced ranges (rows) calibrated with data from the native range or the introduced range (columns). In bold, best model calibration for each range.

\begin{tabular}{|c|c|c|c|c|}
\hline \multirow[b]{3}{*}{ Model for } & \multicolumn{4}{|c|}{ Calibrated with } \\
\hline & \multicolumn{2}{|c|}{ Native range } & \multicolumn{2}{|c|}{ Introduced range } \\
\hline & Mean SE & SD SE & Mean SE & SD SE \\
\hline Native range & 0.67 & 1.67 & 5.78 & 6.35 \\
\hline Introduced range & 2.64 & 4.17 & 1.48 & 2.55 \\
\hline
\end{tabular}

showed slightly higher suitability (Fig. 5). In contrast, habitat suitability in the introduced range was significantly higher in disturbed habitats than in natural habitats (Fig. 5, Table S4 in Appendix S1). For several natural habitats, suitability was significantly higher in the native range (i.e. forest, rocky and shrubland), while differences were not significant for the remaining habitats (Fig. 5). On the other hand, for half of the disturbed habitats suitabilities were higher in the introduced range (i.e. agricultural and urban) and the rest showed no significant differences.

\section{Predicting shifts}

Predictions in the introduced range from models calibrated with native range data showed a larger potential distribution for Oxalis than models using only introduced range data (Fig. 6). 


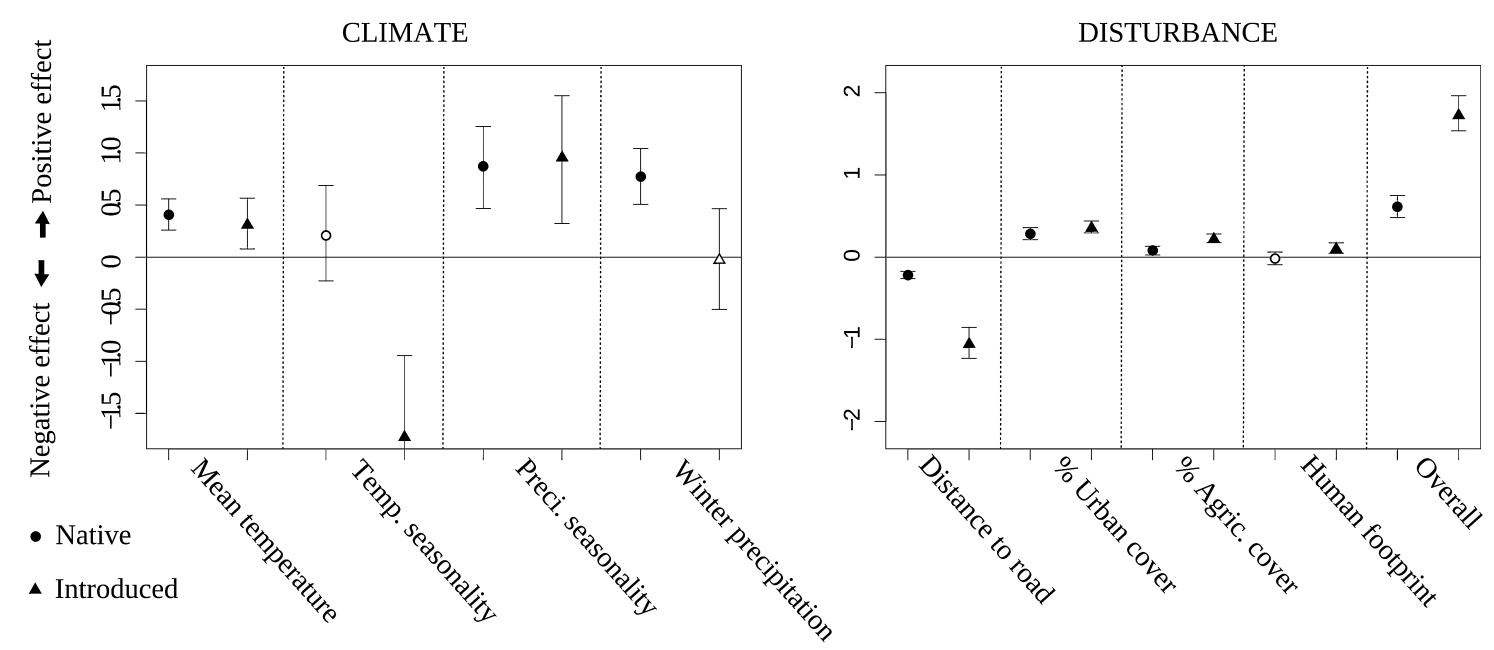

Figure 4 Posterior means (with Bayesian credible intervals) of the coefficients for climate and landscape variables at native (circles) and introduced (triangles) ranges in the generalized linear model explaining the abundance of Oxalis pes-caprae. The 'Overall' coefficient is the absolute sum of the four landscape coefficients. Filled symbols indicate that the $95 \%$ credible interval around the parameter mean values did not include zero (i.e. a significant effect).

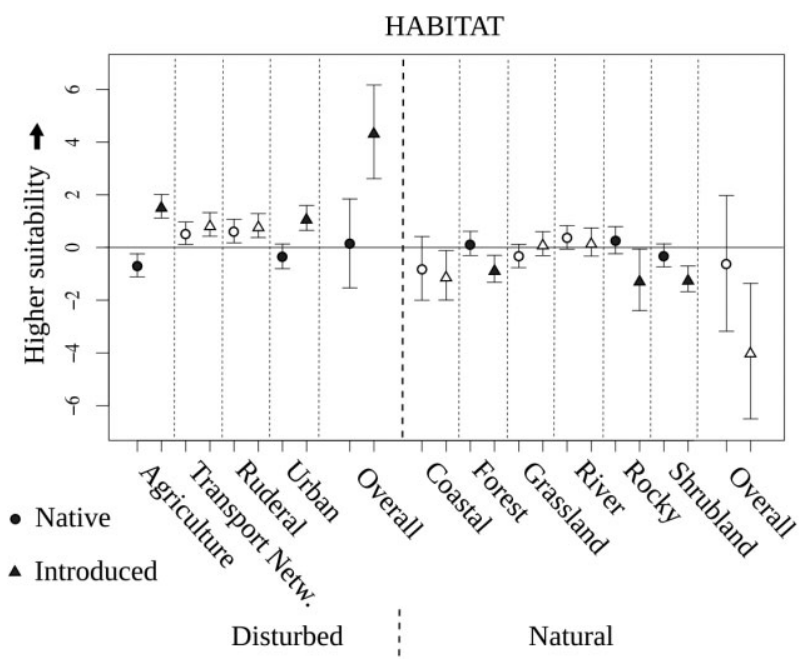

Figure 5 Posterior means (with credible intervals) of the coefficients for habitat type (disturbance at local level) at native (circles) and introduced (triangles) ranges in the generalized linear model explaining the abundance of Oxalis pes-caprae. The 'Overall' coefficients are the sum of the disturbed and natural habitats coefficients, respectively. Filled symbols indicate that the 95\% credible interval around the difference between coefficients of both ranges for each habitat did not include zero (i.e. a significant difference).

However, when considering only predictions for analogous conditions the potential distribution based on native range data was rather small (Fig. 6 \& Fig. S7 in Appendix S1).

\section{DISCUSSION}

Estimating the strength of niche conservatism is extremely important for predicting the spread of introduced species
(Alexander \& Edwards, 2010; Petitpierre et al., 2012). Although most studies of niche shifts consider only broad climatic niches, additional factors such as habitat and disturbance may also affect niche shifts for invasive species. For Oxalis we found similar levels of climatic and disturbance niche shifts between native and introduced ranges, mainly related to different environmental availabilities between ranges and a time lag in the invasion process. Clearly, features other than broad climatic factors are also important for shaping species niches.

\section{Climatic niche shifts}

The observed niche shift of Oxalis towards stronger precipitation seasonality and lower temperatures in the introduced range is most likely a consequence of the availability of climatic conditions in the introduced range that are not present in the native range, rather than rapid evolution. The colder, more seasonal climates in the Mediterranean are simply not available in South Africa. When considering climates available in both ranges, expansion and unfilling metrics were greatly reduced $(<10 \%)$, on a par with percentages found for $86 \%$ of the Holarctic species studied by Petitpierre et al. (2012). Nonetheless, the large expansion observed when considering the whole climatic space suggests that species might have significant plasticity in the niche not expressed in the native range (Alexander \& Edwards, 2010). Similar patterns of expansion linked to high plasticity have been found for species introduced to Australia, with c. $75 \%$ of species studied experiencing novel biomes (Gallagher et al., 2010). These findings have important consequences for analyses of invasion risk because models that consider only the niche in the native range could greatly underestimate the invasion potential of non-native species (Hierro et al., 2009; Jiménez-Valverde et al., 2011).

Half of the climate variables showed similar associations with the abundance of Oxalis in its native and introduced ranges, 

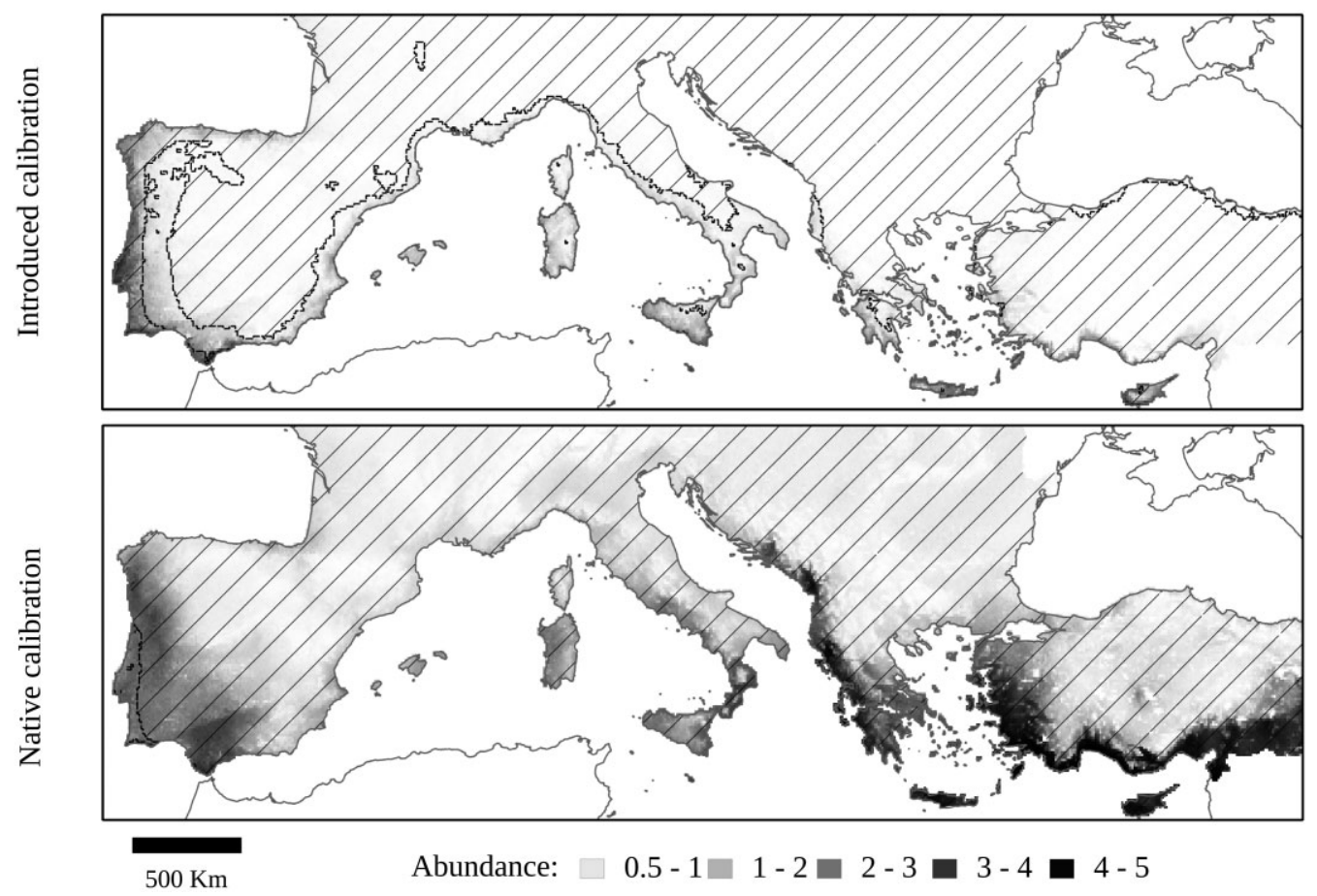

Figure 6 Prediction maps of Oxalis pes-caprae abundance (from absent $=0$ to dominant $=5$ ). Upper part: predictions with the generalized linear model (GLM) calibrated only with data from the introduced range. Lower part: predictions with the GLM calibrated only with data from the native range. The dashed line delimits analogous conditions (positive values of the MESS index) in relation to the calibrated data. A surface crossed with lines indicates non-analogous conditions where models are extrapolating at least in one variable.

suggesting a constant sensitivity to climate across its whole range. However, for the other half of the climate variables, the association was only important in one of the ranges. For example, temperature seasonality was only associated with abundance of Oxalis in the introduced range. The negative association between temperature seasonality and abundance suggests higher abundances close to coastal areas, raising two possible mechanisms. On the one hand it could reflect the original niche in the native range, as Oxalis occupies areas in the native range with relatively weaker seasonality than in the introduced range (Fig. 3). On the other hand, this pattern could be an artefact of the coastal locations of historical introductions and the insufficient time to spread further inland.

Precipitation in winter was only an important correlate of the abundance of Oxalis in the native range. We had expected the same association in the introduced range because the species is a winter-growing geophyte (Ross et al., 2008). Two nonexclusive factors could explain this pattern. First, the species might be associated with artificial water sources in humanaltered areas in the introduced range (personal observation), allowing the species to be less dependent on precipitation regimes. Second, differences could be an artefact of the collinearity between precipitation in winter and precipitation seasonality which occurs only in the introduced range. The different sensitivity of the abundance of Oxalis to climate variables in the two ranges highlights the importance of understanding the biology of invasive species in both their native and introduced ranges rather than developing 'blind' models from the 'best' set of predictors.

\section{Disturbance niche shifts}

Oxalis prefers high levels of disturbance in both its native and introduced ranges. However, we found a clear shift towards higher levels of disturbance in the introduced range, both at landscape and local scales. In other words, Oxalis occupies more natural areas in the native range that remain unoccupied in the introduced range. Similar patterns have been described for other invasive species. For instance, Rhododendron ponticum L. occurs in well-conserved forests in its native range but it is highly invasive in the British Isles where it is especially competitive in disturbed areas (Cross, 1975). Such evidence supports the hypothesis that invasive species may be associated with higher levels of disturbance, especially at the beginning of the invasion process (Hobbs \& Huenneke, 1992; Dietz \& Edwards, 2006). This finding implies that non-native species such as Oxalis could invade more natural habitats as the invasion unfolds (Dietz \& Edwards, 2006). This possibility is further supported by the ecology of Oxalis in its introduced range. Due to the lack of local floral morph diversity in the introduced range, its principal means of reproduction is by bulbils (Castro et al., 2007). This vegetative reproduction could partially explain the shift towards higher levels of disturbance, because disturbance and rapid clonal spread are generally associated (Coffroth \& Lasker, 1998). 
However, the recent discovery of populations with higher floral morph diversity (Castro et al., 2013) and the partial breakdown in the morph-incompatibility system in Portugal (Costa et al., 2014) suggest that the species may be able to take advantage of sexual reproduction and thereby adapt to more natural conditions.

Other factors intrinsic to specific habitats could affect changes in abundance between native and introduced ranges. For instance, Oxalis is more abundant in agricultural habitats in the introduced range, probably due to different crop systems. In southern Europe, the reduction in profitability in modern agriculture (especially olive groves, which are not common in South Africa) promotes systems with lower inputs and weed control, allowing higher abundance of Oxalis compared with similar habitats in South Africa. Biotic interactions could also limit the spread of Oxalis into natural habitats. For instance, Oxalis is a poor competitor against native grasses (Sala et al., 2007). In contrast, Vilà et al. (2006) found similar rates of predation and germination of Oxalis bulbs among ruderal, shrubland and oldfield habitats. Although we lack knowledge about its success in later stages of the recruitment dynamics (Vilà et al., 2006), its 'invasive' character in the native range suggests that the species might be able to expand into more natural habitats, profiting from local disturbances (e.g. forest gaps). This potential expansion could alter succession dynamics in natural habitats, thereby magnifying ecological impacts in the introduced range.

\section{Understanding and modelling niche shifts}

Climatic niche shifts are commonly tested using an aggregation of climate variables in an ordination space (e.g. Gallagher et al., 2010; Petitpierre et al., 2012). Although it is possible to reduce bias in sampling and frequency of environmental conditions by applying smoothing functions (Broennimann et al., 2012), this method still requires a priori knowledge of which variables are most important for determining the range limits of the species. This is a major caveat with this method, because sensitivities to different climatic variables are likely to vary among species. Furthermore we found slightly higher shifts when considering abundance data than with occurrence data, which could partially explain the low shift values found in other studies.

Another frequently used approach is reciprocal distribution modelling in which the potential distribution of a species in the introduced range is predicted from its actual occurrence in the native range and vice versa (Fitzpatrick et al., 2007; Medley, 2010). This technique allows predictions to be mapped in geographical space and accepts categorical variables. Using this approach we have complemented the ordination method by identifying the variables that characterize Oxalis' niche and compared the conservatism in the association of single variables with the species' abundance. Despite its potential, it is important to consider possible bias arising from the selection of the variables (Strubbe et al., 2013) and uncertainties such as that associated with extrapolation into non-analogous conditions (Fitzpatrick \& Hargrove, 2009). Therefore, for shift analyses we advocate a careful selection of biologically relevant variables, the use of both occurrence and abundance data and the combined implementation of ordination and distribution modelling approaches.

The relative merits of quantifying absolute shifts in environmental conditions between ranges, versus shifts within those conditions available in both ranges is currently under debate (Petitpierre et al., 2012). Limiting analyses to conditions that are common to both ranges allows shifts to be interpreted as 'true' niche shifts versus simply the filling of a pre-adapted niche (Petitpierre et al., 2012). However, for invasive species this approach is likely to underestimate invasion risk because introduced ranges often contain environmental conditions that are not available in the native range. Further, our study highlights the importance of defining species niches more broadly than simply by climate because other factors may have important effects on niche shifts (Wiens et al., 2010). For Oxalis, considering only common climatic conditions might underestimate its potential to invade colder, more seasonal and more natural areas. Evidence is mounting that estimations of invasion risk should not assume niche conservatism (Fitzpatrick et al., 2007; Gallagher et al., 2010) but rather incorporate multiple niche dimensions in both native and introduced ranges (Broennimann \& Guisan, 2008; Jiménez-Valverde et al., 2011). Thus, while invasive species offer a unique chance to test basic biogeographic questions about niche shifts, understanding these basic processes also has important consequences for management of biodiversity.

\section{ACKNOWLEDGEMENTS}

We thank A. González, S. Kritzinger-Klopper and K. Rakgalakane for field work assistance in South Africa, and G. Brundu, L. Carta, M. Manca, F. Médail, C. M. Suehs, L. Affre, P. Lambdon and P. E Hulme for providing the European data collected through the project EPIDEMIE (EVK2-CT-200000074). We thank N. E. Zimmermann, B. Petitpierre, J. Levine, J. M. Alexander, C. Küffer and I. Ibáñez for their fruitful comments. Research was funded by the Spanish MINECO projects MONTES (CSD2008-00040) and RIXFUTUR (CGL20097515). D.M.R. acknowledges support from the National Research Foundation (Grant 85417).

\section{REFERENCES}

Affre, L., Suehs, C.M., Charpentier, S., Vilà, M., Brundu, G., Lambdon, P., Traveset, A. \& Hulme, P.E. (2010) Consistency in the habitat degree of invasion for three invasive plant species across Mediterranean islands. Biological Invasions, 12, 25372548.

Alexander, J.M. \& Edwards, P.J. (2010) Limits to the niche and range margins of alien species. Oikos, 119, 1377-1386.

Broennimann, O. \& Guisan, A. (2008) Predicting current and future biological invasions: both native and invaded ranges matter. Biology Letters, 4, 585-589. 
Broennimann, O., Treier, U.A., Müller-Schärer, H., Thuiller, W., Peterson, A.T. \& Guisan, A. (2007) Evidence of climatic niche shift during biological invasion. Ecology Letters, 10, 701709.

Broennimann, O., Fitzpatrick, M.C., Pearman, P.B., Petitpierre, B., Pellissier, L., Yoccoz, N.G., Thuiller, W., Fortin, M.-J., Randin, C., Zimmermann, N.E., Graham, C.H. \& Guisan, A. (2012) Measuring ecological niche overlap from occurrence and spatial environmental data. Global Ecology and Biogeography, 21, 481-497.

Castro, S., Loureiro, J., Santos, C., Ater, M., Ayensa, G. \& Navarro, L. (2007) Distribution of flower morphs, ploidy level and sexual reproduction of the invasive weed Oxalis pescaprae in the western area of the Mediterranean region. Annals of Botany, 99, 507-517.

Castro, S., Ferrero, V., Costa, J., Sousa, A.J., Castro, M., Navarro, L. \& Loureiro, J. (2013) Reproductive strategy of the invasive Oxalis pes-caprae: distribution patterns of floral morphs, ploidy levels and sexual reproduction. Biological Invasions, 15, 1863-1875.

Coffroth, M.A. \& Lasker, H.R. (1998) Population structure of a clonal gorgonian coral: the interplay between clonal reproduction and disturbance. Evolution, 52, 379-393.

Costa, J., Ferrero, V., Loureiro, J., Castro, M., Navarro, L. \& Castro, S. (2014) Sexual reproduction of the pentaploid, short-styled Oxalis pes-caprae allows the production of viable offspring. Plant Biology, 16, 208-214.

Cross, J.R. (1975) Rhododendron ponticum L. Journal of Ecology, 63, 345-364.

D’Austria, S. (1884) Die Balearen-Menorca. Vol. II, Palma de Mallorca, Sa Nostra.

Dietz, H. \& Edwards, P.J. (2006) Recognition that causal processes change during plant invasion helps explain conflicts in evidence. Ecology, 87, 1359-1367.

Elith, J., Kearney, M. \& Phillips, S. (2010) The art of modelling range-shifting species. Methods in Ecology and Evolution, 1, 330-342.

Fitzpatrick, M.C. \& Hargrove, W.W. (2009) The projection of species distribution models and the problem of non-analog climate. Biodiversity and Conservation, 18, 2255-2261.

Fitzpatrick, M.C., Weltzin, J.F., Sanders, N.J. \& Dunn, R.R. (2007) The biogeography of prediction error: why does the introduced range of the fire ant over-predict its native range? Global Ecology and Biogeography, 16, 24-33.

Gallagher, R.V., Beaumont, L.J., Hughes, L. \& Leishman, M.R. (2010) Evidence for climatic niche and biome shifts between native and novel ranges in plant species introduced to Australia. Journal of Ecology, 98, 790-799.

Gassó, N., Thuiller, W., Pino, J. \& Vilà, M. (2012) Potential distribution range of invasive plant species in Spain. NeoBiota, $12,25-40$.

Geerts, S., Moodley, D., Gaertner, M., Le Roux, J.J., McGeoch, M.A., Muofhe, C., Richardson, D.M. \& Wilson, J.R.U. (2013) The absence of fire can cause a lag phase: the invasion dynamics of Banksia ericifolia (Proteaceae). Austral Ecology, 38, 931941.
Gimeno, I., Vilà, M. \& Hulme, P.E. (2006) Are islands more susceptible to plant invasion than continents? A test using Oxalis pes-caprae L. in the western Mediterranean. Journal of Biogeography, 33, 1559-1565.

González-Moreno, P., Pino, J., Carreras, D., Basnou, C., Fernández-Rebollar, I. \& Vilà, M. (2013) Quantifying the landscape influence on plant invasions in Mediterranean coastal habitats. Landscape Ecology, 28, 891-903.

Guisan, A., Petitpierre, B., Broennimann, O., Daehler, C. \& Kueffer, C. (2014) Unifying niche shift studies: insights from biological invasions. Trends in Ecology and Evolution, 29, 260269.

Hierro, J.L., Eren, Ö., Khetsuriani, L., Diaconu, A., Török, K., Montesinos, D., Andonian, K., Kikodze, D., Janoian, L., Villarreal, D., Estanga-Mollica, M.E. \& Callaway, R.M. (2009) Germination responses of an invasive species in native and non-native ranges. Oikos, 118, 529-538.

Hijmans, R.J., Cameron, S.E., Parra, J.L., Jones, P.G. \& Jarvis, A. (2005) Very high resolution interpolated climate surfaces for global land areas. International Journal of Climatology, 25, 1965-1978.

Hobbs, R.J. \& Huenneke, L.F. (1992) Disturbance, diversity, and invasion: implications for conservation. Conservation Biology, 6, 324-337.

Jiménez-Valverde, A., Peterson, A.T., Soberón, J., Overton, J.M., Aragón, P. \& Lobo, J.M. (2011) Use of niche models in invasive species risk assessments. Biological Invasions, 13, 27852797.

Lauzeral, C., Leprieur, F., Beauchard, O., Duron, Q., Oberdorff, T. \& Brosse, S. (2011) Identifying climatic niche shifts using coarse-grained occurrence data: a test with non-native freshwater fish. Global Ecology and Biogeography, 20, 407414.

Lunn, D., Spiegelhalter, D., Thomas, A. \& Best, N. (2009) The BUGS project: evolution, critique, and future directions. Statistics in Medicine, 28, 3049-3067.

Medley, K.A. (2010) Niche shifts during the global invasion of the Asian tiger mosquito, Aedes albopictus Skuse (Culicidae), revealed by reciprocal distribution models. Global Ecology and Biogeography, 19, 122-133.

Neis, P., Zielstra, D. \& Zipf, A. (2011) The street network evolution of crowdsourced maps: openStreetMap in Germany 2007-2011. Future Internet, 4, 1-21.

Olson, D.M., Dinerstein, E., Wikramanayake, E.D., Burgess, N.D., Powell, G.V.N., Underwood, E.C., D’amico, J.A., Itoua, I., Strand, H.E., Morrison, J.C., Loucks, C.J., Allnutt, T.F., Ricketts, T.H., Kura, Y., Lamoreux, J.F., Wettengel, W.W., Hedao, P. \& Kassem, K.R. (2001) Terrestrial Ecoregions of the World: A New Map of Life on Earth. BioScience, 51, 933-938.

Ornduff, R. (1987) Reproductive systems and chromosome races of Oxalis pes-caprae L. and their bearing on the genesis of a noxious weed. Annals of the Missouri Botanical Garden, 74, 79-84.

Peterson, A.T., Papes, M. \& Kluza, D.A. (2003) Predicting the potential invasive distributions of four alien plant species in North America. Weed Science, 51, 863-868. 
Petitpierre, B., Kueffer, C., Broennimann, O., Randin, C., Daehler, C. \& Guisan, A. (2012) Climatic niche shifts are rare among terrestrial plant invaders. Science, 335, 1344-1348.

Ross, L.C., Lambdon, P.W. \& Hulme, P.E. (2008) Disentangling the roles of climate, propagule pressure and land use on the current and potential elevational distribution of the invasive weed Oxalis pes-caprae L. on Crete. Perspectives in Plant Ecology, Evolution and Systematics, 10, 251-258.

Sala, A., Verdaguer, D. \& Vilà, M. (2007) Sensitivity of the invasive geophyte Oxalis pes-caprae to nutrient availability and competition. Annals of Botany, 99, 637-645.

Spiegelhalter, D.J., Best, N.G., Carlin, B.P. \& Van Der Linde, A. (2002) Bayesian measures of model complexity and fit. Journal of the Royal Statistical Society: Series B (Statistical Methodology), 64, 583-639.

Strubbe, D., Broennimann, O., Chiron, F. \& Matthysen, E. (2013) Niche conservatism in non-native birds in Europe: niche unfilling rather than niche expansion. Global Ecology and Biogeography, 22, 962-970.

Thuiller, W., Richardson, D.M., Pyšek, P., Midgley, G.F., Hughes, G.O. \& Rouget, M. (2005) Niche-based modelling as a tool for predicting the risk of alien plant invasions at a global scale. Global Change Biology, 11, 2234-2250.

Urban, M.C., Phillips, B.L., Skelly, D.K. \& Shine, R. (2007) The cane toad's (Chaunus $[$ Bufo] marinus) increasing ability to invade Australia is revealed by a dynamically updated range model. Proceedings of the Royal Society B: Biological Sciences, 274, 1413-1419.

Vilà, M., Bartomeus, I., Gimeno, I., Traveset, A. \& Moragues, E. (2006) Demography of the invasive geophyte Oxalis pescaprae across a Mediterranean island. Annals of Botany, 97, 1055-1062.

Vilà, M., Pino, J. \& Font, X. (2007) Regional assessment of plant invasions across different habitat types. Journal of Vegetation Science, 18, 35-42.

WCS \& CIESIN (2005) Last of the Wild Project, version 2, 2005 (LWP-2): global human footprint database. NASA Socioeconomic Data and Applications Center (SEDAC), Palisades, NY. Available at: http://dx.doi.org/10.7927/H4M61H5F

Weber, E. (2003) Invasive plant species of the world: a reference guide to environmental weeds. CABI, Wallingford.

Wiens, J.J., Ackerly, D.D., Allen, A.P., Anacker, B.L., Buckley, L.B., Cornell, H.V., Damschen, E.I., Jonathan Davies, T., Grytnes, J.-A., Harrison, S.P., Hawkins, B.A., Holt, R.D., McCain, C.M. \& Stephens, P.R. (2010) Niche conservatism as an emerging principle in ecology and conservation biology. Ecology Letters, 13, 1310-1324.

\section{SUPPORTING INFORMATION}

Additional supporting information may be found in the online version of this article at the publisher's web-site.
Appendix S1 Complementary tables and figures.

Figure S1 Principal component analysis plot of the global Mediterranean space showing the contribution of each climatic variable to the first two axes.

Figure S2 Principal component analysis plots of the sampled space (Mediterranean Basin and South Africa) showing the contribution of climate and landscape variables to the first two axes. Figure S3 Detailed global climatic niche of Oxalis in native and introduced ranges.

Figure S4 Climatic and disturbance niche shifts of Oxalis based on occurrence data of the Mediterranean analysis.

Figure S5 Detailed climatic and disturbance niche of Oxalis in native and introduced ranges corresponding to the Mediterranean analysis.

Figure S6 Posterior means of the coefficients for climate and landscape variables at native and introduced ranges in the generalized linear model explaining the abundance of Oxalis only from road sampling data.

Figure S7 Map of MESS values for predictions in the introduced range.

Table S1 Person's correlation coefficient of the association among all variables used in the analyses.

Table S2 Pearson's correlation coefficient of the association between abundance of Oxalis and land-cover variables at 250, 500 and $1000 \mathrm{~m}$.

Table S3 Differences between native and introduced ranges for each climate and landscape coefficient.

Table S4 Differences of the posterior means of the coefficients for habitat at native and introduced ranges in the generalized linear model explaining the abundance of Oxalis.

Appendix S2 Modelling details and OpenBugs code.

\section{BIOSKETCH}

Pablo González-Moreno is a doctoral candidate at EBD-CSIC and works on the macroecology of plant invasions (http://www.glezmoreno.es).

Author contributions: P.G., M.V., J.D. and D.M.R. conceived the study; M.V. and P.G. designed and conducted the field surveys, P.G. analysed the data and led the writing; M.V., J.D. and D.M.R. commented on analyses and contributed to writing the manuscript.

Editor: Niklaus Zimmerman 